\title{
Aeroacoustic Simulation for NASA CC3 Centrifugal Compressor Operating at off Design Condition
}

\author{
Mohamed Alqaradawi ${ }^{1}$, Ibrahim Shahin ${ }^{2}$, Mohamed Gadala $^{3}$ and Osama Badr ${ }^{4}$ \\ ${ }_{2}^{1}$ Mechanical and Industrial Engineering Department Collage of Engineering, Qatar University, Doha (2713), Qatar. \\ ${ }^{2}$ Mechanical and Industrial Engineering Department, Collage of Engineering, Qatar University, Doha (2713), Qatar. \\ ${ }^{2}$ Mechanical Engineering Department, Shoubra faculty of Engineering, Benha University, Egypt. \\ ${ }^{3}$ Mechanical Engineering Department, UBC-University of British Columbia. Vancouver, BC, Canada. \\ ${ }^{4}$ Mechanical Engineering Department, the French University in Egypt, Al-Shorouk City, New Cairo.
}

\begin{abstract}
This paper covers the characterization of the acoustic noise and the unsteady flow field of a high speed centrifugal compressor NASA CC3. In order to accurately predict the noise, all analyses are carried out through the use of Large Eddy Simulation and Ffowcs Williams-Hawkings model for noise prediction. The relative effect of hub cavity on flow characteristics and sound levels is investigated, for a compressor stage with a total pressure ratio equal to 4 , working from surge to near choke condition. In comparison with the experimental results from literature, the predicted compressor performance and flow field are predicted well. The hub cavity flow effect on the compressor aeroacoustic generated noise is shown in the paper. The unsteady static pressure and sound pressure levels are compared not only at different location but also for design and off design operating points. The internal flow results inside the hub cavity are presented at surge, design and near choke points. The conclusion is that the cavity effect of the centrifugal compressor cannot be ignored in the numerical prediction of aerodynamic generated noise. The impeller back plate of the rotor experiences a strong pressure fluctuation, which is maxima at the impeller outer radius for all operating point, but higher pressure values at the surge point.
\end{abstract}

\section{Introduction}

The aero-acoustic analysis of the centrifugal compressor has become urgent not only due to demands requirements for environment-friendly products [1] but also to prevent compressors from mechanical failure [2]. If acoustics resonances occur within centrifugal compressor, the pressure fluctuations may reach very high levels. Even though compressor parts are designed for high cycle fatigue; this would jeopardizing the safe operation of the compressor and the facility. Rotating stall induces large vibratory stresses in the blading of compressors and is therefore undesirable for structural reasons although the compressor may continue to give acceptable performance [3]. Surge consists of large-amplitude oscillations of the flow through the entire compressor which also produces large oscillations in compressor delivery pressure [4]. Extensive studies have been developed on the unsteady flow in centrifugal compressors focusing on rotating surge phenomena. Mckain and Holbrook defined both aerodynamic and manufacturing design details for $4: 1$ pressure ratio single stage centrifugal compressor [5]. The data published by Skoch, et al. [6] included experimental measurements using a Laser-Doppler Anemometer to obtain flow-field velocity measurements in a centrifugal impeller. Wernet, et al. [7] conducted pressure transducer measurements to capture the transient response of a compressor prior to rotating stall. During this investigation, Digital Particle Image Velocimetry was applied to capture transient velocity and pressure measurements during compressor surge. In fact, reviewing existing studies on aero-acoustic interaction indicates that centrifugal compressors have received less care than their axial compressor counterparts and especially in the unsteady aerodynamics flow. Interference generated in axial compressors has received much attention, due to the strict patterns in the aeroengine industry because of its aeronautical use and public demand for aircraft with lower noise [8], [9]. Hanson [10] showed that under certain inlet and exit conditions, an acoustic mode could get trapped between blade rows leading to amplification and higher frequency propagation. Mengle [11] analysed the physical aspects of spinning acoustic modes produced by blade vibration. Besides, the theoretical development is conducted to understand and anticipate the frequency spectra observed in the stationary and rotating reference frames.

Raitor and Neise [12] measured aero acoustic performance and investigated the sources and mechanisms of sound generation of the spectral components governing the overall noise level of centrifugal compressors. The sound generation 
mechanisms of centrifugal compressors are well described for low compressor speeds. Recently, Konig et al. [13] presented two case studies on shrouded impeller under high cycle fatigue, one based on a 2D impeller stage with a vaned diffuser, the other on a $3 \mathrm{D}$ impeller stage with a vaneless diffuser. The authors presented a coupling mechanism between impeller vibration mode, a Tyler-Sofrin excitation resulting from impeller/stator interaction, and secondary flow cavity acoustic modes.

Petry et al. [14], [15] conducted experimental study with unsteady pressure instrumentation. Acoustic resonances in the side cavities of the centrifugal compressor stage have been characterized. The results showed that a cavity acoustic mode is excited when the Tyler-Sofrin mode and the cavity eigen-mode have the same frequency and circumferential harmonics. Richards et al. [16] conducted experimental and unsteady CFD investigation for complex aero-acoustic interaction between the impeller and the upstream and downstream return channel vanes. However, the CFD study of Richards et al. [16]-[14] conducted only on blade row interactions and not including the hub and shroud cavities acoustic. Comprehensive descriptions and investigations of the flow between rotating and stationary discs, can be found in the work of Tuliszka-Sznitko [17], [18], Gauthier et al. [19], Pellé [20] and Serre [21], but most of these studies neglected the complicated flow out from the impeller region and the unsteady flow during the surge event. Medic et al. [22] performed a numerical study using Large-eddy simulation (LES) with wall-adapting local eddy-viscosity (WALE) subgrid scale model to explain the complex turbulent flow in NACA CC3 centrifugal impeller, obtained with LES, Reynolds Averaged NavierStokes (RANS) turbulence models and hybrid RANS/LES approaches. The present paper is a continuation of a previously presented study of Shahin et al. [23] and focuses on the influence of the unsteady pressure and swirling flow in the hub side cavity on the acoustic levels. Since cavity effects are shown to be significant, in this research, a model of compressor with and without the hub cavity is proposed. Centrifugal compressor hub cavity flow simulation has been neglected by a lot of previous works, which discuss the aeroacoustic simulation for centrifugal compressors [16], [24] and [25].

\section{Test Case Presentations}

For the simulations performed in this work, we consider the well-studied NASA CC3 compressor that was tried over many years at the Small Engine Components Test Facility at NASA Glenn Research Center [5]-[7]. The computational model consists of three parts; the inlet pipe with an inlet bell at its exit, rotating impeller and Vaneless diffuser with impeller hub side cavity followed by shaft seal, shown in Fig. 1. A straight pipe with length 5 times the inlet diameter is attached at the compressor inlet to insure fully developed flow at the inlet. The duct wall is included in the numerical implementation to get very accurate predictions of noise field. The impeller is followed by a Vaneless diffuser with an annular radial-to- axial bend. The axial bend is extended to minimize the effect of outlet boundary conditions on the flow inside the diffuser. Fig. 1 shows the basic geometry and simulation domain for the moving and stationary parts. The computational grid generated for one passage is shown in Fig. 2 and the flow path through impeller, diffuser and hub cavity is shown in Fig. 3. The compressor stage with no cavity is also shown in Fig. 3; the impeller exit flow is completely go through the diffuser.

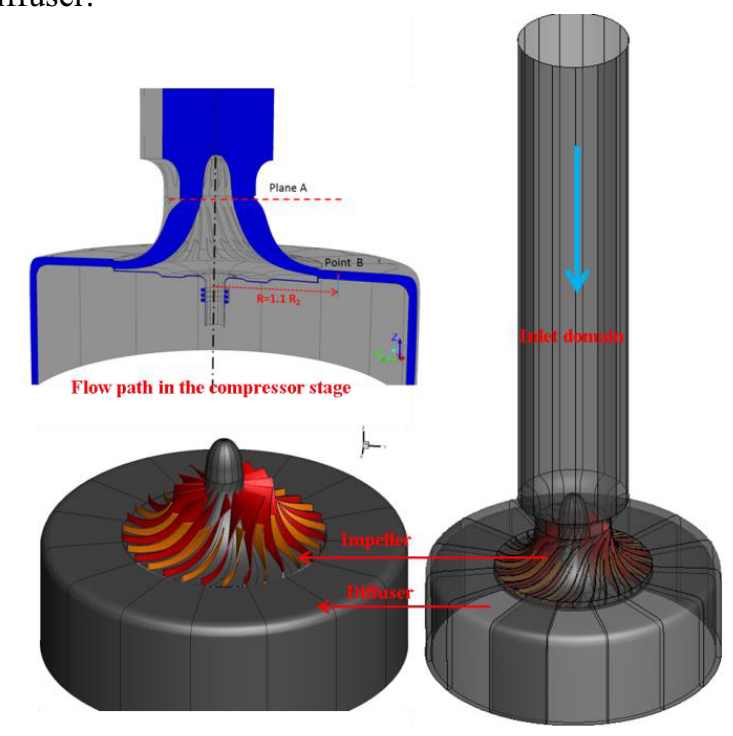

Figure 1. The geometry of simulated parts

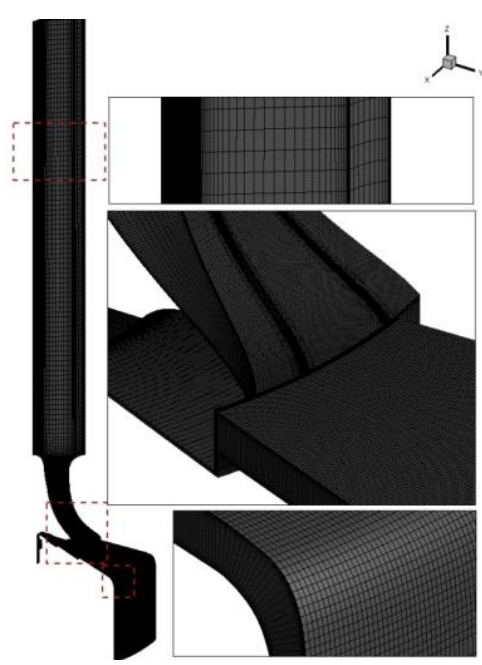

Figure 2. Computational domain grid "3.23 Million of nodes"

\section{Numerical procedures}

\subsection{Mathematical model}

Three-dimensional, Navier-Stokes equations are solved for compressible flow. The sliding mesh is used for simulating the impeller rotation, in which the multiple cell zones are connected to each other through interface boundary condition. As the mesh motion is updated in time, the interfaces are likewise updated to reflect the new positions of each zone [26]. 


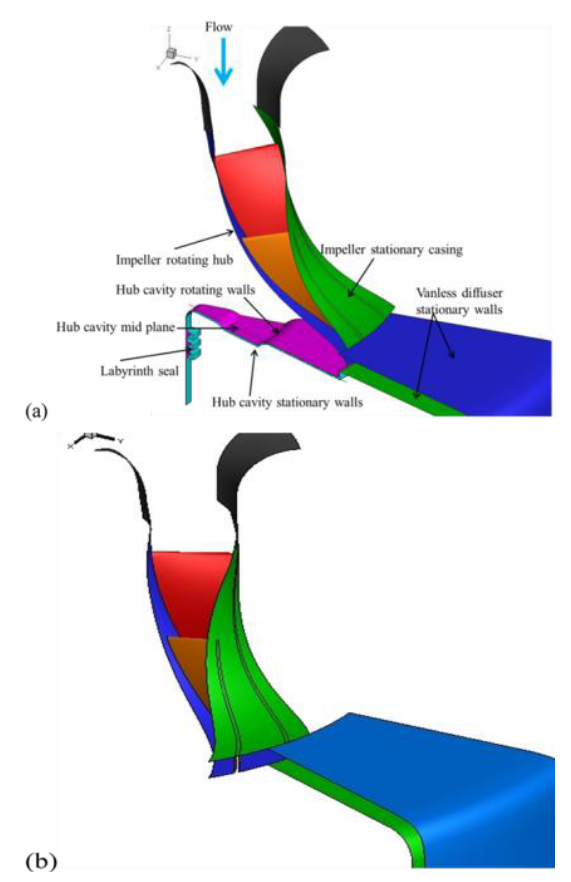

Figure 3. Flow path in the compressor stage for: (a) with cavity, (b) without cavity

The unsteady large scale structures and separation zones are the main characteristics of the flow in centrifugal compressor; therefore the large eddy simulation (LES) approach is used. In LES, large eddies are resolved directly, while small eddies are modelled with sub-grid scale model. A major role of the small scales is to dissipate the turbulent energy that is transferred from the larger scales to the smaller ones through the energy cascade. In the LES approach, the separation between the resolved and unresolved scales is achieved by low-pass spatial filtering of the governing equations. The subgrid-scale stresses resulting from the filtering operation are unknown, and require modelling. In the present case, subgrid Smagorinsky-Lilly model is used [27].

The frequency domain approach of Ffowcs WilliamsHawkings formulation applicable to the computed domain is chosen as the governing equation for the noise prediction [28], which has been used also by [24], [25] for predicting the aerodynamic noise in a centrifugal compressor. The ANSYS-FLUENT program with parallel processing, version 14.5 [26] was used to solve the filtered governing equations, which are discretized by the finite volume method. For the spatial discretization, a second order upwind scheme was used. The second order implicit formulation was used for the temporal discretization. In order to resolve the relevant fluctuations, high grid resolution with enough small time steps were employed to have the Courant number smaller than one to be consistent with the flow properties which, for this case, give a time step of 6.119 e- 6 sec. The unsteady calculations are performed with a time-step size so that the impeller mesh turns less than $1^{\circ}$ per time step, this time-step is sufficient to accurately resolve pressure spectra within human hearing range. In LES it is desirable to make sure that no physical information can propagate further than one grid cell at one time step. This gives a coupling between the temporal and the spatial discretization of the problem. This condition is expressed in terms the Courant Friedrichs Lewy (CFL) condition, which states that the Courant number must be less than one.

\subsection{Boundary conditions}

The mass flow inlet boundary condition is used at the inlet of the pipe with atmospheric pressure and $288 \mathrm{~K}$ and pressure outlet boundary condition at the domain outlet. The pressure outlet boundary condition is specified at the seal exit which equal to atmospheric pressure. Rotating periodic boundary condition is used. Wall boundary condition with no slip velocity is employed on walls. The rotating cavity face has the same rotational speed of the impeller, while the fixed cavity face has zero speed. The impeller casing wall has zero absolute speed.

Table 1. Operating conditions and unsteady simulation parameters

\begin{tabular}{ll|c}
\hline Condition & & Value \\
\hline Design Inlet pressure & $\mathrm{bar}$ & 1.013 \\
Design pressure ratio & & $4: 1$ \\
Inlet temperature & $\mathrm{K}$ & 288 \\
Design mass flow rate & $\mathrm{lbm} / \mathrm{sec}$ & 10 \\
Surge mass flow rate & $\mathrm{lbm} / \mathrm{sec}$ & 7.2 \\
Rotational Speed & $\mathrm{rpm}$ & 21789 \\
Total simulated flow time & $\mathrm{sec}$ & 0.175 \\
Time step & $\mathrm{sec}$ & $6.119 \mathrm{e}-6$ \\
Number of time steps & & 28600 \\
Number of iteration per time step & 20 \\
Hardware operating time per case day & 75 \\
Number of simulated surge cycles & 2 \\
\hline
\end{tabular}

The multi-block structured hexahedral mesh created using ICEM CFD 14.5 blocking tools is used for meshing computational domain, which consist of one blade sector. In order to capture the boundary layer, which is important for compressor flows, the mesh is refined at the walls. The blade tip clearance is resolved with 10 cells in the radial direction. The grid is concentrated near the walls and the measured $\mathrm{y}+$ is less than 1 . The grid is enlarged by a ratio of $5 \%$ as it moved further away from the walls. Operating conditions and unsteady simulation parameters are listed in Table 1.

\subsection{Mesh sensitivity study and numerical model validation}

In order to ensure the accuracy of present numerical solution, a careful check for present numerical model was conducted. In this section, the computed data are compared with measured data to validate the developed model for both the performance map data and the internal flow velocity in the vaneless diffuser. Careful check for grid independence was also conducted; three numbers of computational nodes were used 3.23, 4.36 and 6.451 million of nodes. The absolute velocities normalized by impeller tip speed at design flow rate with different 
number of computational nodes are shown in Fig. 4, for Skotch et al. [6] experimental results and present CFD. The present CFD results for mesh 3.23 and 4.36 million of nodes under estimate the velocity profile after the impeller outlet, while the results with 6.451 million nodes agree well with experimental data from [6].

It is clear that the tip leakage flow is well detected, so the grid with 6.451 million nodes was used for all the studies conducted in the present simulation. Overall, results compare favourably, although the present CFD simulation predicts slightly lower velocities near the hub and shroud walls of the vaneless diffuser. These differences may be attributable to the smooth and the adiabatic walls assumption made during the simulation.
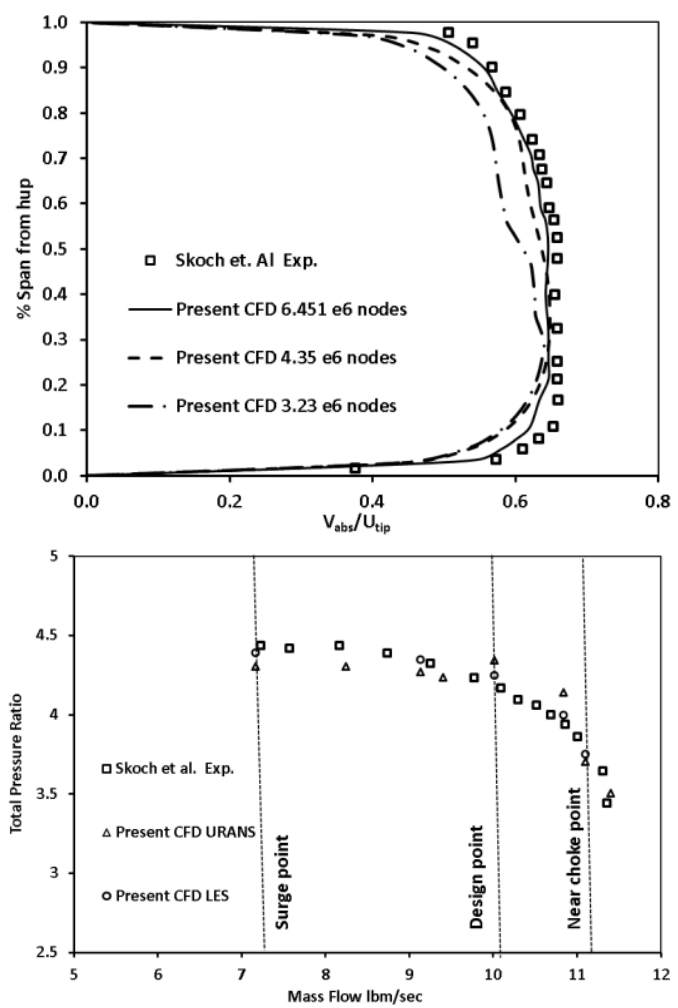

Figure 4. Comparison of measured data [6] and present simulation: (Upper) Velocity in the vaneless diffuser for different computational number of nodes, (Lower) Stage performance map.

\section{Results and discussion}

In the following sections, the flow physics in the mid span and near tip plans at surge, design and near choke points are presented. Also the flow field in the hub cavity region will be analysed at these flow rates, so as to explain the reason for high SPL for lower flow rates and also for the compressor with cavity case.

\subsection{Compressor impeller flow}

In this section, the mass flow signals are monitored with the flow time. These signals are acquired at three different operating points; surge, design and near choke conditions. Fig. 5 presents the time resolved mass flow rate at the impeller inlet, and the fluctuation in the mass flow is increased by moving to lower mass flow rate. Near the surge point noticeably higher amplitude fluctuations were observed. Also, the surge event is characterized not only by high mass flow fluctuation at impeller inlet, but also by the flow reversal at the front of the impeller. This can be observed in Fig. 5a in which the mass flow at impeller inlet is monitored with the flow time. On the other hand, no flow reversal was observed in the impeller front when operating at the design flow rate Fig. $5 \mathrm{~b}$, but only a small fluctuation in the mass flow is observed with no change in the flow direction. Fig. 6 shows the tip leakage vortex structure, the tip leakage vortices are clearly found rolling up from the leading edge and the corresponding stream lines also indicated that. The tip leakage vortex of the full-blade seems to be moving from the main blade suction side towards but not reaching the next main blade pressure side.
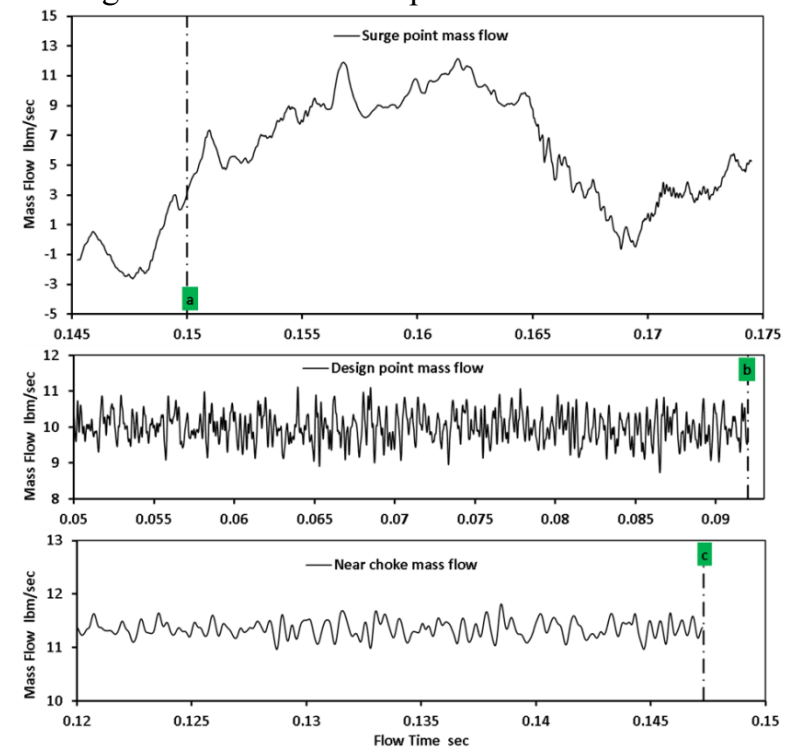

Figure 5. Mass flow with time monitored at impeller inlet

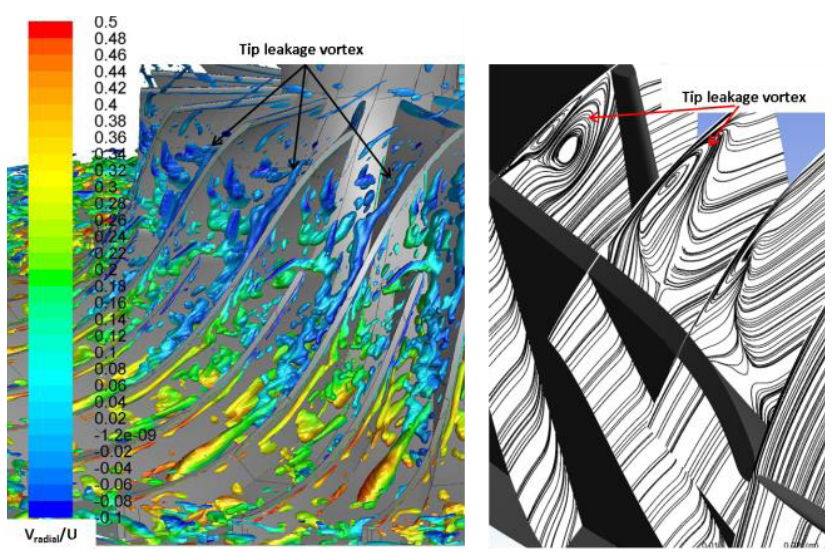

Figure 6. Tip leakage vortex structure by: (Left) Normalized QCriterion equal 0.1 colored by radial velocity, ( Right) Stream lines at different meridional sections at the impeller inducer.

\subsection{Vaneless diffuser flow}

Fig. 7a shows the vaneless diffuser core flow during surge condition and it is clear the vaneless diffusers have 
rotating instability that is similar to rotating stall. The rotating instabilities, characterized by a core of outward radial velocity followed by a zone of inward radial velocity, are quite well identifiable. Fig. $7 b$ shows the absolute iso-velocity values corresponding to the design operating flow conditions. The flow pattern in the diffuser inlet is characterized by the tip leakage vortex and the jet/wake structure, which resulting from the interaction of the impeller secondary flows including the tip leakage flow. Fig. 8 shows the instantaneous static pressure at 0.5 span and 0.95 span planes, for design, surge and choke flow conditions. The pressure values are higher at 0.95 span planes more than that at 0.5 spans due to the tip leakage flow from the impeller outlet. The pressure is increased gradually from the impeller inlet to the diffuser outlet, but the pressure fluctuation is higher for design point more near chock operating point, the highest fluctuation is detected at the surge point. The rotating flow instability in the diffuser and flow reversal in the impeller are the main reasons for higher pressure fluctuation.
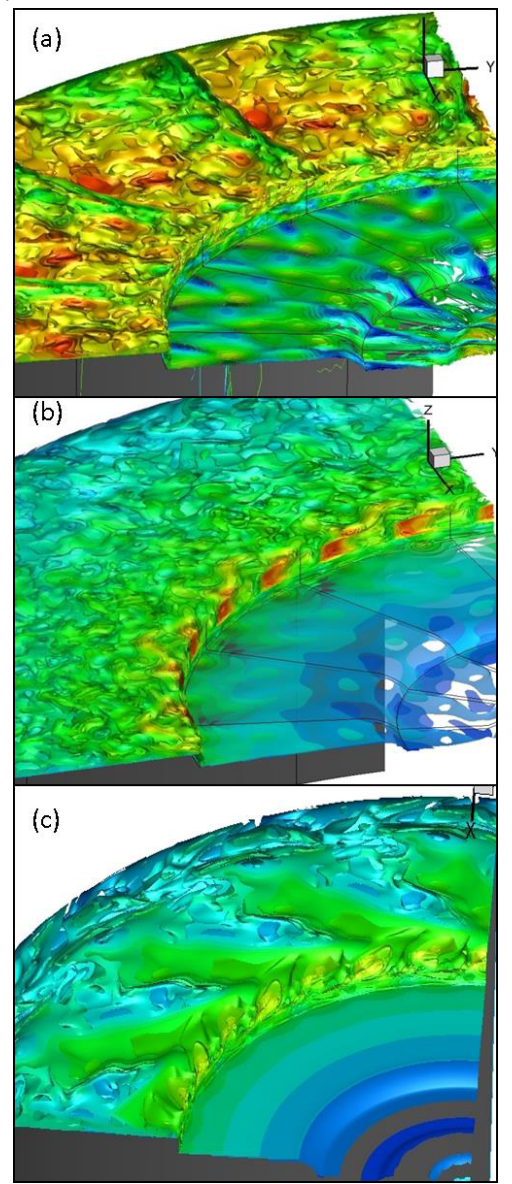

Figure 7. Dimensionless instantaneous velocity ' $\mathrm{V} / \mathrm{U} 2$ ' isovalues in diffuser and hub cavity at (a) Surge (b) Design (c) Near choke

The instantaneous radial velocity is shown in Fig. 9 for different operating flow rats. The aerodynamic behaviour in the diffuser is strongly changed by moving from design operating point towards left and right in the compressor performance map. For the surge operating condition, the low momentum zone appears along the diffuser hub side and continues downstream up to the diffuser end. This is due to the impeller outlet fluid removal through the back face cavity, and the flow is aspirated through the cavity. Separation zones near the casing just after the impeller outlet are detected as a result from the impeller tip leakage flow and the flow tends to separate close to the diffuser end due to the diffusion inside the diffuser. Close to choke operating point, the influence of hub cavity leakage flows is much smaller and lower separated regions are detected. The main flow is pushed towards the casing side, as a result from the swirling flow coming to the diffuser domain from the hub cavity. Consequently, a low momentum flow close to the hub appeared due to the removal of the mainstream flow into the hub cavity flow. The radial velocity for the no cavity case is shown in Fig 9.d, it is clear that radial velocity is increase than that in Fig. 9.b as no flow extracted through the cavity, also no flow separation is detected at the diffuser hub due to the absence of the cavity. The absence of flow separation near the cavity will results in low acoustic noise when compared with case with cavity.

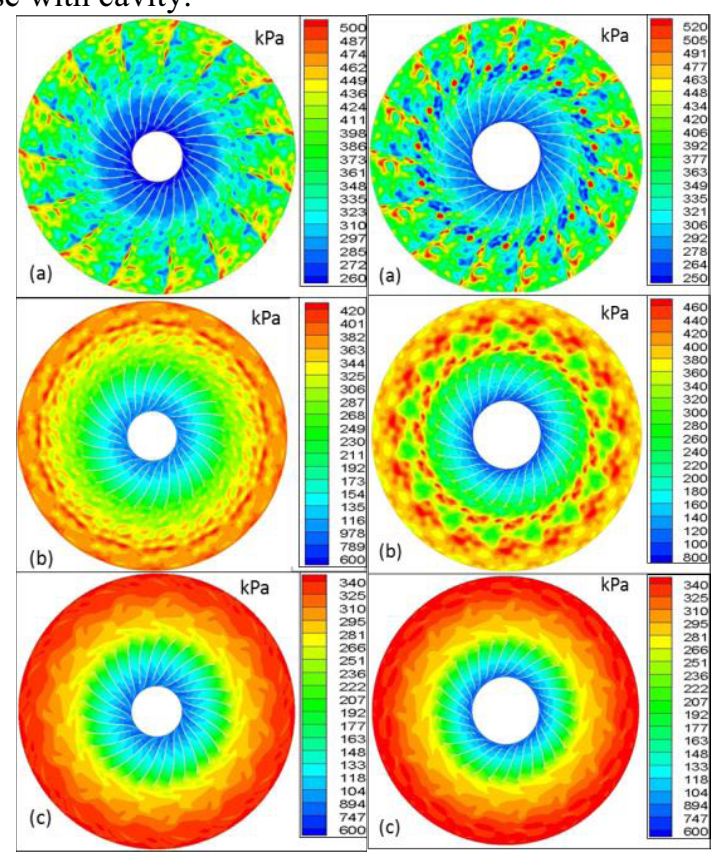

Figure 8. Instantaneous Static pressure in $\mathrm{kPa}$ at (a) Surge (b) Design (c)Near choke ( Left: 0.5 span, Right: 0.95 span)

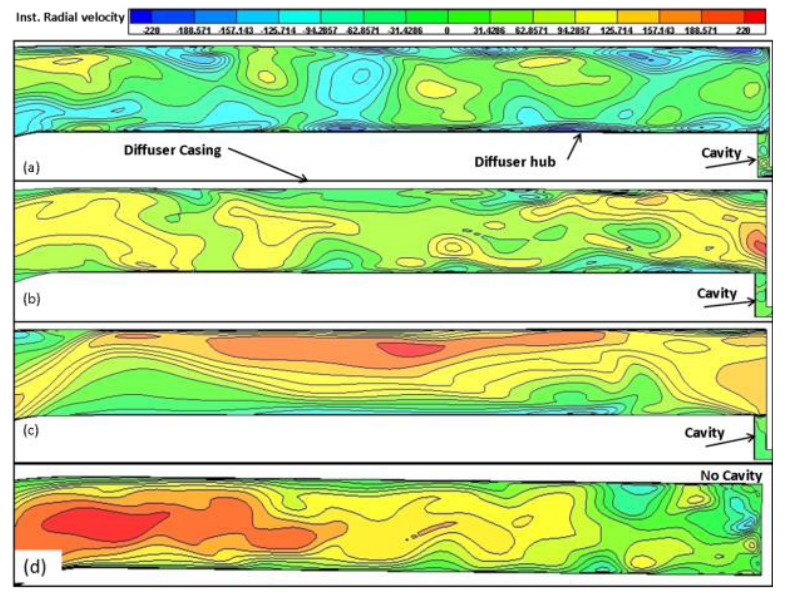

Figure 9. Instantaneous radial velocity at diffuser for (a) Surge (b) Design (c) Near choke and (d) No cavity case at design point 


\subsection{Hub cavity flow}

Fig. 10 shows the leakage flow through the hub cavity as a percentage of the operating mass flow during the simulation time. The amount of mass flow passing through the hub cavity depends on pressure recovery and total pressure losses of downstream stationary parts. Changing the flow from the design operating point towards surge and choke affects the amount of flow through the cavity. The hub cavity flow rate is increased as surge condition, as the pressure loss is increased in the diffuser and flow blockage. Leakage flow in the hub cavity tends to decrease at near choke conditions as the pressure recovery in the diffuser is decreased. The amount of flow passing through the cavities depends, for certain geometry, on the combined effects of pressure recovery and total pressure losses of downstream stationary components for the hub one. Moving from design operating point to lower or higher flow rates point, the amount of flow through the cavity tends to increase and decrease, respectively.

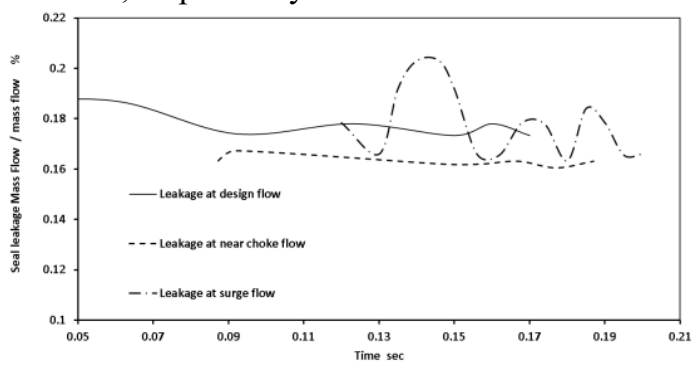

Figure 10. Leakage flow through the hub cavity with time

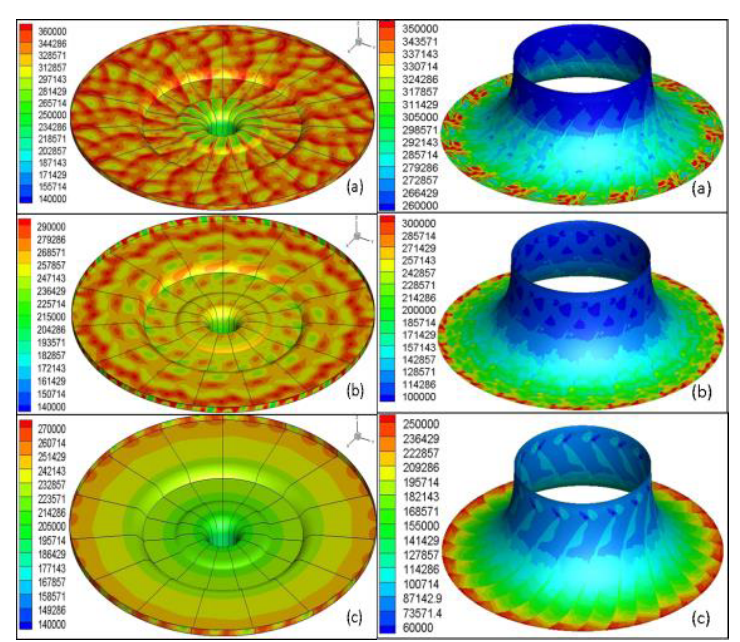

Figure 11. Instantaneous static pressure in $\mathrm{Pa}$ on the impeller casing side at (a) Surge (b) Design (c) Near choke. (Left: Impeller back plat, Right: Impeller shroud)

The generation of rotating pressure patterns due to the interaction between rotating and stationary components is shown in Fig. 11. The instantaneous distribution of such pressure pattern is shown for the impeller back plate and the casing. It can be seen that the impeller back plate of the rotor experiences a strong pressure fluctuation. The pressure maxima are located at the impeller outer radius all operating point, but higher pressure values are generated for the surge point. The plots show that instantaneous static pressure is greatest near the impeller trailing edge, with a significant increase in loading values when moving from near choke towards the surge point. The tip leakage vortices at the impeller exit plane is one of reasons causes a noticed increase of noise downstream of the impeller.

The fluctuated flow during the surge point also causes a fluctuation on the cavity flow, which results in rotating flow instability inside the cavity and an increase for the static pressure fluctuation. The unsteady pressures in the cavities are much higher than that on the compressor casing.

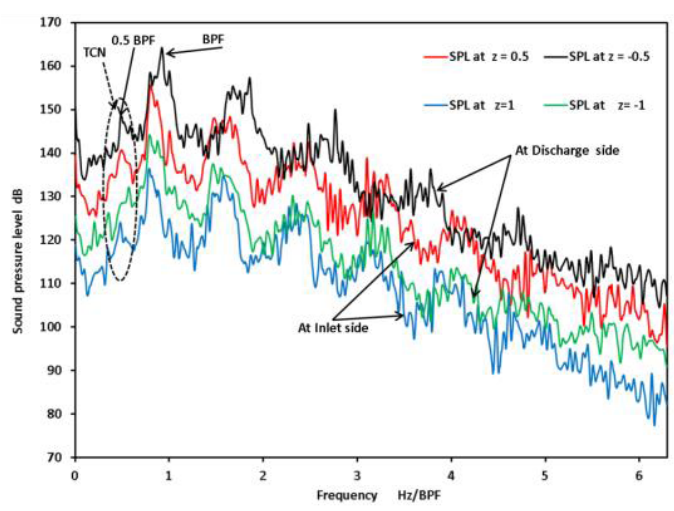

Figure 12. Sound pressure spectra for no cavity case at different locations

\subsection{Sound pressure level signal analysis}

The acoustic pressure signals were captured at the inlet side and discharge side of the compressor. The SPL is monitored and captured during one complete surge cycles for the surge operating conditions. These signals were computed in time domain and then converted to the frequency domain using the FFT algorithm. Sound pressure receiver locations relative to compressor parts are shown in Fig. 12, All the receivers are located on the compressor rotation axis and $\mathrm{z}$ represents the distance on compressor axis measured from the compressor nose which represents the zero value for $z$, positive values of $z$ corresponds to the inlet side while negative $\mathrm{z}$ corresponds to the discharge side. The receivers on the discharge side are located outside the CFD domain, and this is agreed by ANSYS FLUENT [26]. In Fig. 12 the sound pressure spectra monitored during simulation at compressor suction and discharge receivers are plotted. The $\mathrm{x}$ axis represents the frequency normalized by the BPF (blade passing frequency, $10895 \mathrm{~Hz}$ ). The BPF sound level component is seen obviously in the spectrum with high magnitudes. With increasing BPF harmonic order, the levels of the blade tone harmonics become smaller, and they decay at high frequency levels. The receiver position has a big effect on the SPL levels. As the receiver becomes close to the impeller outlet, the more noticeable are the BPF and its harmonics. Other noise component is observed at about half the BPF; this component magnitude is lower than the BPF level. It is produced by the tip leakage flow through the gap between the compressor casing and the impeller blade tips "tip clearance noise" (TCN), as observed by [10]. The sound 
pressure level spectra monitored on the compressor discharge side is higher than that measured in the inlet side, because noise components generated at the interface between impeller exit and stationary parts " diffuser inlet " could not transferred through the complicated flow fields in the rotor blade channels to the compressor inlet side.

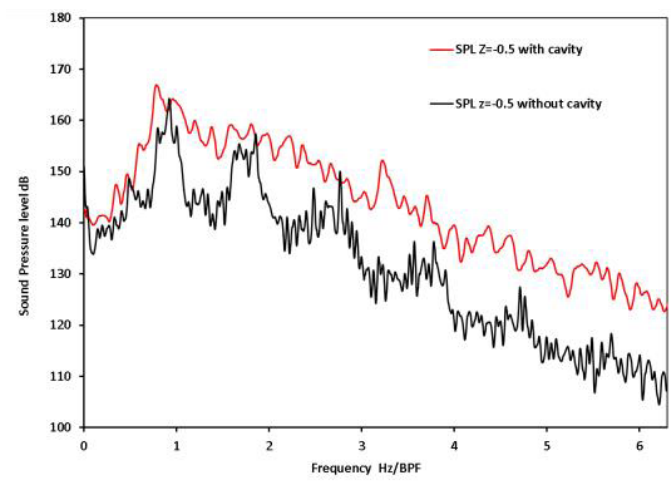

Figure 13. Sound pressure spectra for case with and without cavity $\mathrm{z}=-0.5$, for design point.
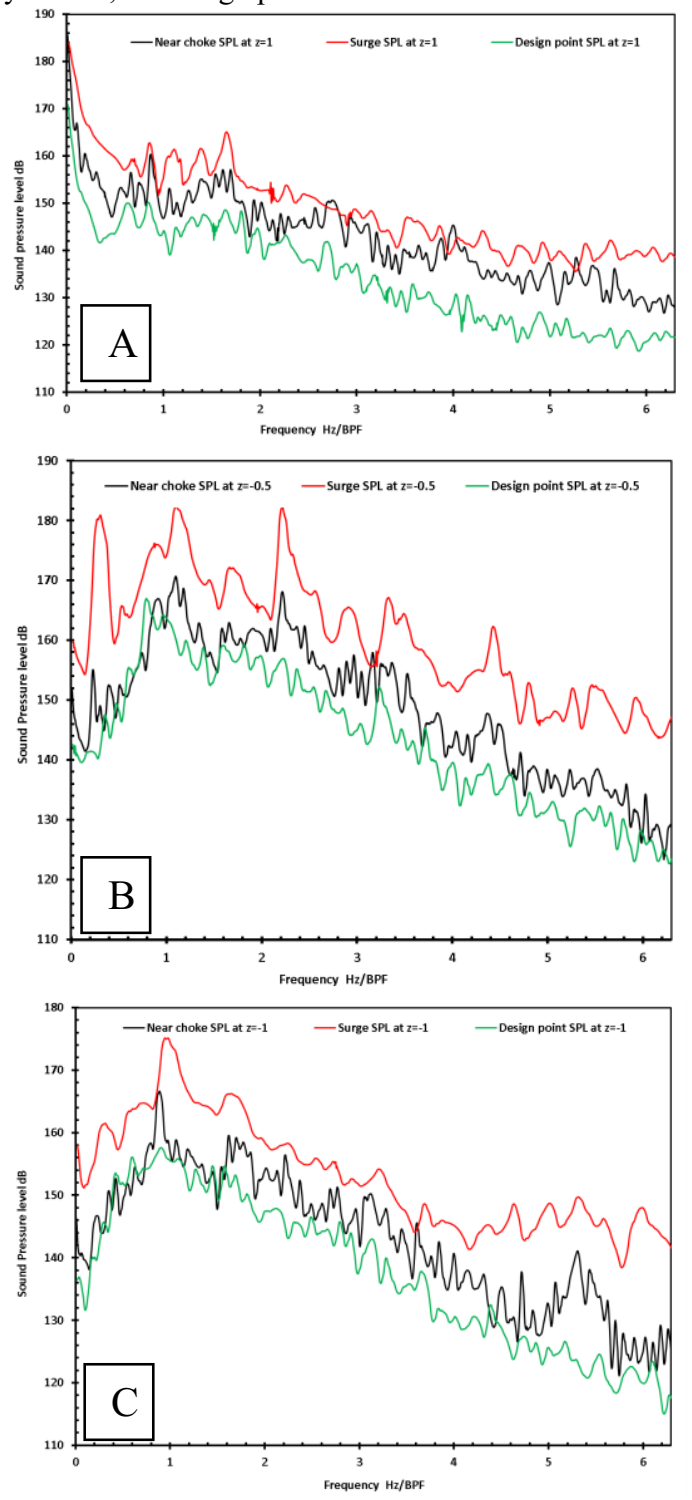

Figure 14. Sound pressure spectra for operating point for compressor with cavity at (a) $z=1$, (b) $z=-5$ and (c) $z=-1$
Sound pressure spectra for the compressor with and without hub cavity are compared in Fig. 13. The SPL for the cavity case is higher than that without cavity. This difference is increased at higher frequencies. The flow swirling and high rotating pressure generated in the hub cavity is the cause of this difference, which has been shown in this paper before (Section 5.3) that the flow in the hub cavity is characterized by high swirling flow. Fig. 14 compares the SPL spectra of the compressor working from surging to near choking conditions under full speed; the compressor produces sound with significant changes as the mass flow varies. The SPL at surge condition is the maximum for all receiver positions.

\section{Conclusions}

In this paper, we examined the aerodynamic and aero acoustics performance of a transonic centrifugal compressor working from surge to near choke conditions. The effect of hub cavity on the flow characteristics and sound levels is investigated numerically. Three dimensional simulations for the compressor with and without hub cavity are performed. The entire compressible flow inside the centrifugal compressor stage is simulated through the use of LES, and Ffowcs Williams-Hawkings model for noise prediction. The SPL for the cavity case is more eminent than that without cavity, this difference increases at higher frequencies. The flow swirling and high rotating pressure generated in the hub cavity may be the reason of this conflict.

The results also demonstrate that, the vaneless diffuser during surge have a rotating instability that is similar to rotating stall. The hub cavity flow rate is increased at surge condition, as the pressure losses is increased in the diffuser and flow blockage. The impeller back plate of the rotor experiences a strong pressure fluctuation higher than that detected on the shroud, which results in a generation of axial force on the impeller. The pressure maxima are located at the junction of the impeller and diffuser for all operating point, but higher pressure values are brought forth from the surge point. The propagation of rotating pressure patterns in the hub cavity is due to the interaction between rotating and stationary parts. Finally, The research done here gives a details on the hub side cavity effect on the acoustic sources for the centrifugal compressor and shows the importance of simulating the impeller cavities when deal with the compressor acoustics. The cavities of the centrifugal compressor should be included in the mathematical prediction of the noise.

\section{Acknowledgments}

This publication was made possible by NPRP grant No. 4-651- 2-242 from the Qatar National Research Fund (a member of Qatar Foundation). The statements made herein are solely the responsibility of the authors. 


\section{References}

1. Gottlob, D. Regulations for community noise. Noise News International, 3(4), 223-236, (1995)

2. König, S., Petry, N., \& Wagner, N. G. Aeroacoustic Phenomena in High-Pressure Centrifugal Compressors-A Possible Root Cause for Impeller Failures. In Proceedings of the 38th Turbomachinery Symposium, pp. 103-121, (2009)

3. Stenning, A. H. Rotating stall and surge. Journal of Fluids Engineering, 102(1), 14-20, (1980)

4. Emmons, H. W., Pearson, C. E., \& Grant, H. P. Compressor surge and stall propagation. Trans. ASME, 77(4), 455-469, (1955)

5. McKain, T. F., \& Holbrook, G. J. Coordinates for a high performance 4: 1 pressure ratio centrifugal compressor. Lewis Research Center, NASA-23268, (1997)

6. Skoch, G. J., Prahst, P. S., Wernet, M. P., Wood, J. R., \& Strazisar, A. J. Laser anemometer measurements of the flow field in a 4: 1 pressure ratio centrifugal impeller. In ASME 1997 International Gas Turbine and Aero engine Congress and Exhibition American Society of Mechanical Engineers, pp. V001T03A049-V001T03A049, (June 1997)

7. Wernet, M. P., Bright, M. M., \& Skoch, G. J. An investigation of surge in a high-speed centrifugal compressor using digital PIV. Journal of Turbomachinery, 123(2), 418-428, (2001)

8. Holste, F., \& Neise, W. Noise source identification in a propfan model by means of acoustical near field measurements. Journal of Sound and Vibration, 203(4), 641-665, (1997)

9. Tyler, J. M., \& Sofrin, T. G. Axial flow compressor noise studies (No. 620532). SAE Technical Paper, (1962)

10. Hanson, D. B. Mode trapping in coupled 2D cascades - acoustic and aerodynamic results. AIAA paper, (93-4417), (1993)

11. Mengle, V. G. Acoustic spectra and detection of vibrating rotor blades, including row-to-row interference. In AIAA, Space Programs and Technologies Conference, vol. 1, (October 1990)

12. Raitor, T., \& Neise, W. Sound generation in centrifugal compressors. Journal of Sound and Vibration, 314(3), 738-756, (2008)

13. Konig, S. Acoustic eigenmodes in the side cavities of centrifugal compressors. In ASME Turbo Expo 2009: Power for Land, Sea, and Air American Society of Mechanical Engineers, pp. 547-557, (January 2009)

14. Petry, N., Benra, F. K., \& Koenig, S. Experimental study of acoustic resonances in the side cavities of a high-pressure centrifugal compressor excited by rotor/stator interaction. In ASME Turbo Expo 2010: Power for Land, Sea, and Air. American Society of Mechanical Engineers, pp. 2339-2351, (October 2010)
15. Petry, N., König, S., \& Benra, F. K. Influence of the Swirling Flow in the Side Cavities of a Coupled Blade Row Interactions. Journal of Turbomachinery, 134(6), (2013)

16. Richards, S. K., Ramakrishnan, K., Shieh, C. M., Moyroud, F., Picavet, A., Ballarini, V., \& Michelassi, V. Unsteady Acoustic Forcing on an Impeller Due to, (2012)

17. Tuliszka-Sznitko, E., \& Zieliński, A. DNS/LES of Transitional Flow in Rotating Cavity. International Journal of Transport Phenomena, 10(3), (2008).

18. Tuliszka-Sznitko, E., Zielinski, A., \& Majchrowski, W. Large eddy simulation of non-isothermal flow in rotor/stator cavity. Ichmt Digital Library Online, (2009)

19. Gauthier, G., Gondret, P., Moisy, F., \& Rabaud, M. Instabilities in the flow between co-and counterrotating disks. Journal of Fluid Mechanics, 473: 1-21, (2002)

20. Pellé, J., \& Harmand, S.. Heat transfer measurements in an opened rotor-stator system air-gap. Experimental thermal and fluid science, 31(3), 165180, (2007)

21. Serre, E., Crespo Del Arco, E., \& Bontoux, P. Annular and spiral patterns in flows between rotating and stationary discs. Journal of Fluid Mechanics, 434: 65-100, (2001)

22. Medic, G., Feng, J., Chen, L., \& Sharma, O.. Towards Large-Eddy Simulation of Turbulent Flow in a Centrifugal Impeller. In ASME 2011 Turbo Expo: Turbine Technical Conference and Exposition, American Society of Mechanical Engineers, pp. 2057-2066, (January 2011)

23. Shahin, I., Gadala, M., Alqaradawi, M., \& Badr, O.. Unsteady CFD Simulation for High Speed Centrifugal Compressor Operating Near Surge. In ASME Turbo Expo 2014: Turbine Technical Conference and Exposition (pp. V02DT44A045V02DT44A045). American Society of Mechanical Engineers, (June 2014)

24. Wang, P., \& Zangeneh, M. Aerodynamic and Aeroacoustic Optimization of a Transonic Centrifugal Compressor. In ASME Turbo Expo 2014: Turbine Technical Conference and Exposition (pp. V02AT41A012-V02AT41A012). American Society of Mechanical Engineers, (June 2014)

25. Sun, H., Shin, H., \& Lee, S. Analysis and optimization of aerodynamic noise in a centrifugal compressor. Journal of sound and Vibration, 289(4), 999-1018, (2006)

26. ANSYS, "Academic Research Release 14.5, Theory Manual," ANSYS, Irvine CA. (2012)

27. Meneveau, C., \& Katz, J. Scale-invariance and turbulence models for large-eddy simulation. Annual Review of Fluid Mechanics, 32(1), 1-32, (2000)

28. Ffowcs Williams, J.E. and Hawkings, D.L.. Sound generated by turbulence and surfaces in arbitrary motion. Philos Trans R Soc A, 264:321-342, (1969) 